\title{
Laboratory restructuring and service support for infection control programs
}

Laboratory restructuring is dramatically changing the face of clinical microbiology laboratories across Canada. To a large extent, changes within the laboratory sector are being driven by severe funding cuts to healthcare. Laboratory restructuring began in Alberta in 1992, and due to a 30\% to 40\% overall decrease in funding of laboratory services in Calgary, a new service delivery model arose. Calgary Laboratory Services (CLS) is a new, publicly funded company that has been contracted to provide diagnostic testing for the region since November 1 , 1996. CLS is a partnership between the Calgary Regional Health Authority (CRHA) which represent hospitals and a private company, MDS-Kasper Laboratories. Many similar types of public/private laboratory mergers are occurring across the country.

At the same time that funding cuts were occurring, there has been an increased demand for specialized laboratory services for infection control support in our region. Several key factors are driving this demand as follows.

- Antibiotic-resistant organism (ARO) surveillance -

We are now living in a new era where as a consequence of widespread antibiotic use, drug resistance has emerged as a global public health crisis in the past few years. Many different types of bacteria that commonly cause human infections have rapidly developed a variety of mechanisms to resist the attack by antibiotics. The consequence is that antibiotics, which once seemed invincible against common bacterial infections, are rapidly losing their ability to treat a wide range of community-acquired infections, including gonorhea and infections caused by Streptococcus pneumoniae (ie, otitis media, sinusistis, pneumonia, meningitis). Much media attention has also been given to hospital outbreaks caused by multiply resistant bacteria such as methicillin-resistant Staphlococcus aureus (MRSA) and vancomycin-resistant enterococci (VRE). The treatment of serious infections caused by Gram-negative organisms has also become more difficult due to acquisition of multiple resistance to many commonly used, previously effective drugs. Emergence of these and other types of antibiotic resistance in pathogenic bacterial organisms has and will likely continue to increase the laboratory services that must be provided.

- Regionalization of the infection control programs Expansion of infection control activities into regional programs has had a substantial effect on the volume of specialized microbiology services that are needed. Before regionalization, hospital microbiology laboratories provided services to on-site infection control program activities. Regional infection control programs expanded surveillance activities to include nursing homes and acute care clinics in the community, such as out-patient renal dialysis and surgery.

- Centralization of acute care services - Healthcare restructuring in Calgary has made it necessary to transfer patients between acute care hospitals because of the centralization of major clinical services in a single facility. For example, all neurosurgical services are located at the Foothills Medical Centre. To prevent the potential spread of antibiotic-resistant organisms being carried by high risk patients who are transferred from one facility to another, regional infection control policies outline that nose and/or rectal samples must be tested by the microbiology laboratory to detect colonization/infection with an ARO.

- Changing patient care environment - The likelihood of isolating an ARO from patients being cared for in the community has also increased because of the earlier discharge of sick, high risk patients from hospital to home care. Identification of patients carrying an ARO is important so that appropriate isolation measures can be immediately started on admission to the hospital. Similarly, elderly patients from long term care facilities may also become colonized and/or infected with an ARO during their acute hospital stay.

Baseline laboratory costs for infection control support must now be estimated for healthcare regions. Adequate laboratory funding must be provided to offer the full range of services required by an excellent infection control/ARO surveillance program. Calculation of an adequate level of laboratory funding for delivery of infection control services is difficult because there are little baseline data available. Previous budgets most often did not account for these tests in a separate cost centre. Without these financial data, one can only estimate what the laboratory costs will be of a fully regionalized infection control program. Administrators must also recognize that new funding has never been established for the increased laboratory costs of expanded ARO surveillance activities. Because ARO screening service needs will likely increase in proportion to the prevalence of particular organisms in a given geographic region, laboratory funding must be reassessed on a regular basis as service needs change. Monies should be provided for ARO-surveillance based upon the rate and volume of testing performed in the past year. It is also important that administrative processes be put in place for funding new tests in a capped laboratory budget system such as exists in Alberta. Development and evaluation of new procedures for rapid ARO 


\section{LETTERS TO THE EDITOR}

Continued from page 126

detection will be essential to maintain the success of infection prevention efforts at a minimal healthcare cost.

Laboratory expenses are not the only costs that must be accounted to infection control programs. Other key program components and their costs need to be analyzed along with establishing accurate costing data for laboratory services. Wherever possible, a financial balance sheet is needed for institutional and/or regional savings that result from the effective prevention of individual episodes of patient infection and outbreaks. Accurate Canadian data are needed for this side of the balance sheet to justify more easily increased expenditures for all of the costs of running an excellent regional infection control program. Rapidly changing economic forces within Canadian healthcare should make this type of research a priority for ourselves and decision-makers.

Deirdre L Church MD PhD FRCPC Clinical Leader, Microbiology Calgary Laboratory Services

Calgary, Alberta 


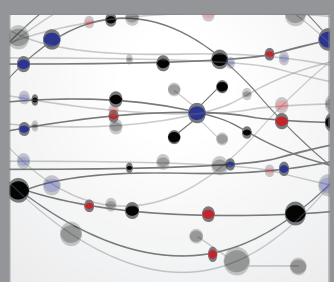

The Scientific World Journal
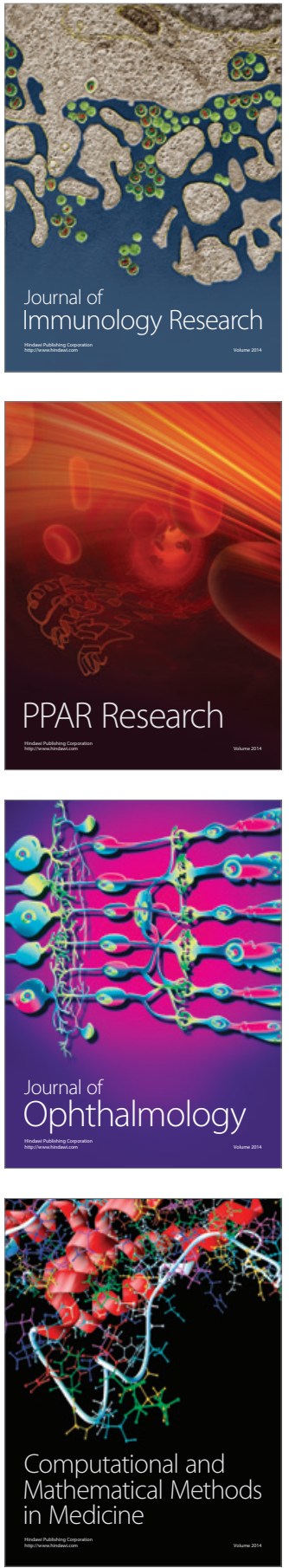

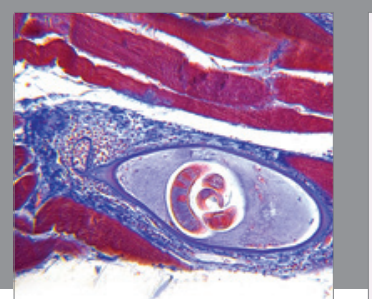

Gastroenterology Research and Practice

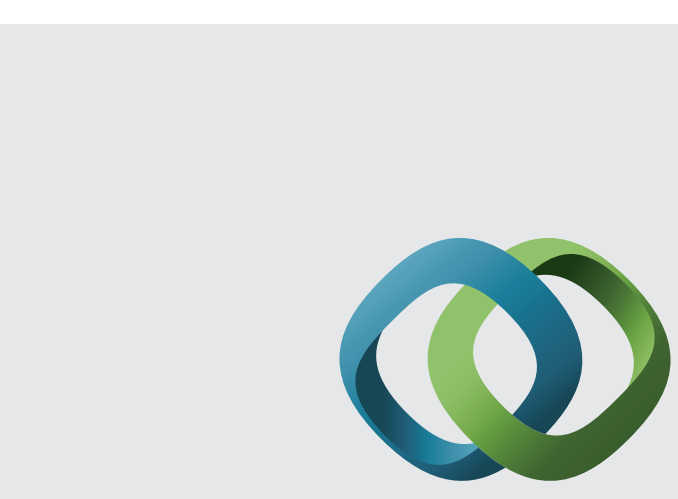

\section{Hindawi}

Submit your manuscripts at

http://www.hindawi.com
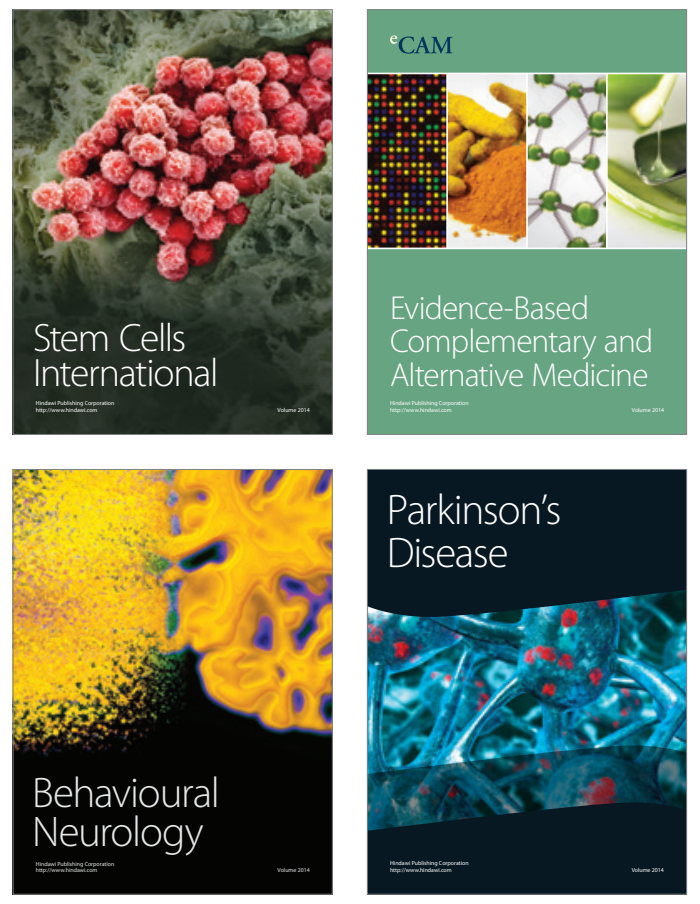
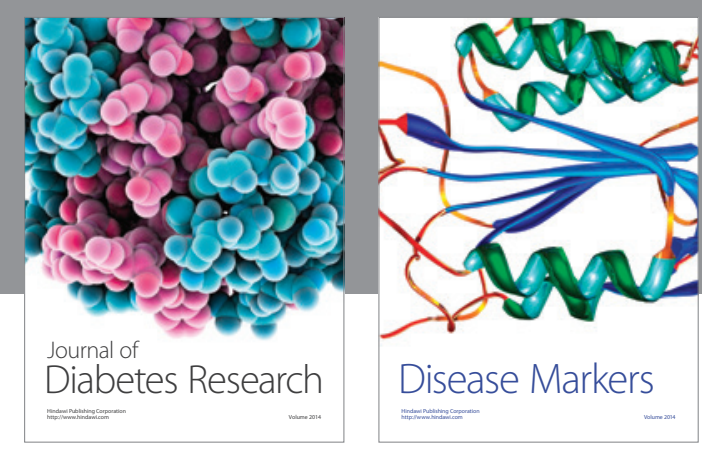

Disease Markers
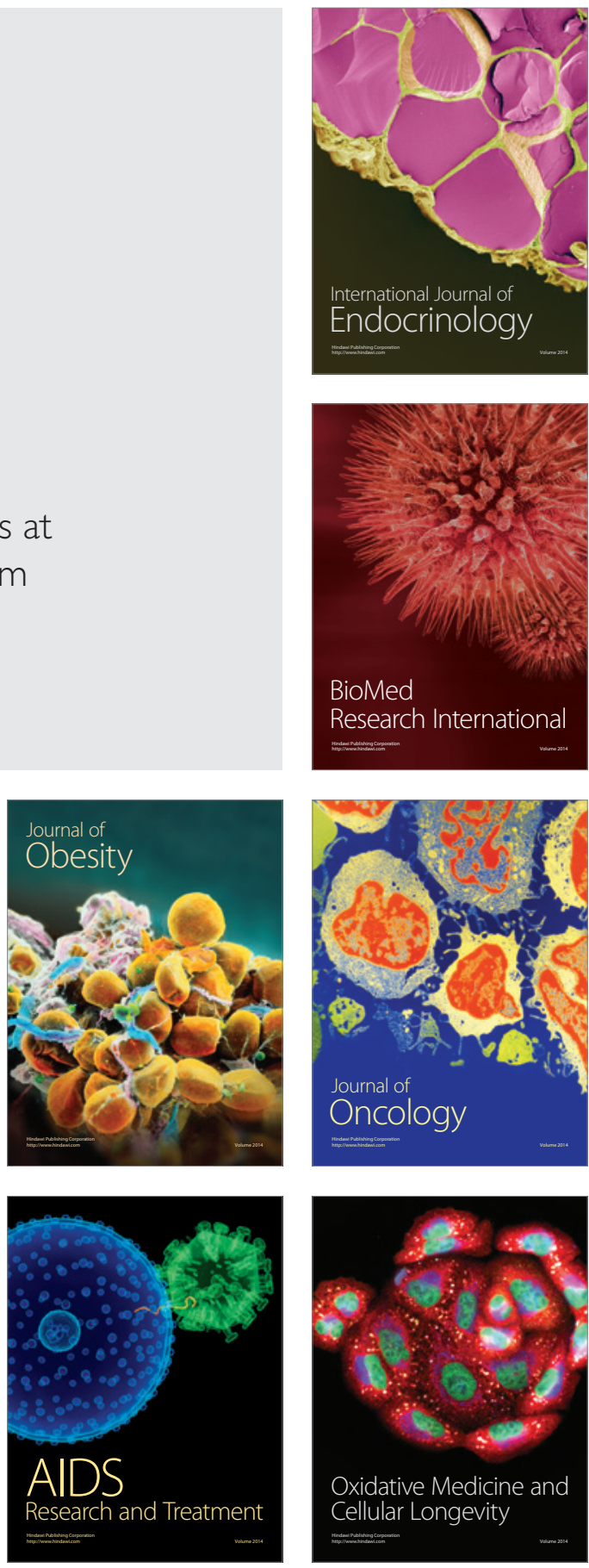